\title{
Acknowledgement to Reviewers of JMSE in 2016
}

\author{
JMSE Editorial Office \\ Published: 12 January 2017 \\ MDPI AG, St. Alban-Anlage 66, 4052 Basel, Switzerland; jmse@mdpi.com
}

The editors of JMSE would like to express their sincere gratitude to the following reviewers for assessing manuscripts in 2016.

We greatly appreciate the contribution of expert reviewers, which is crucial to the journal's editorial process. We aim to recognize reviewer contributions through several mechanisms, of which the annual publication of reviewer names is one. Reviewers receive a voucher entitling them to a discount on their next MDPI publication and can download a certificate of recognition directly from our submission system. Additionally, reviewers can sign up to the service Publons (https://publons.com) to receive recognition. Of course, in these initiatives we are careful not to compromise reviewer confidentiality. Many reviewers see their work as a voluntary and often unseen part of their role as researchers. We are grateful to the time reviewers donate to our journals and the contribution they make.

If you are interested in becoming a reviewer for JMSE, see the link at the bottom of the webpage http://www.mdpi.com/reviewers.

The following reviewed for JMSE in 2016:

Adamo, Maria

Ainslie, Michael

Akbar, Muhammad

Alberello, Alberto

Alizad, Karim

Allgeyer, Sebastien

Alves, Tiago

Andersen, Poul

Aretxabaleta, Alfredo

Ayca, Aykut

Backer, Annelies

Barrera, Carlos

Barron, Mace

Béchet, Quentin

Bedri, Zeinab

Bergdahl, Lars

Bergillos, Rafael

Bergstrom, Per

Birch, Gavin

Blackman, Donna

Blanco Chao, Ramon

Blanton, Brian

Blossier, Brice

Boavida, Joana

Boleslaw Grzegorz, Giren

Bongiorni, Lucia
Bowen, James

Brandon, Christine M.

Briggs, Tiffany

Brune, David

Buscombe, Daniel

Butler, Edward

Cavagnaro, Robert J.

Cerrano, Carlos

Certain, Raphael

Chi, Kai Hsien

Chou, Loke Ming

Chow, Yi-chih

Chu, Dezhang

Colagrossi, Andrea

Congestri, Roberta

Corinaldesi, Cinzia

Costa, Sandra

Cremades Ugarte, Javier

Cristini, Paul

Dahl, Peter

Dalyander, Patricia

De Andres, Adrian

De Mendoza, Francesco Paladini

Del Castillo, Carlos E.

Deng, Bing

Dill, Nathan 
Dolores Esteban, Maria

Donelan, Mark

Dreier, Norman

Dresback, Kendra

Dunford, Adam

Dunlop, Erin S.

El-Gindy, Moustafa

Fairley, Iain

Falcieri, Francesco

Ferrari, Michele

Ferrarin, Christian

Ferreira, Bruno

Feuillassier, Lionel

Finelli, Christopher M.

Fleming, Jason

Flüh, Ernst

Foeth, Evert-Jan

Fulton, Mike

Gabiña, Gorka

Gallien, Timu W.

García, Sergio

Garcia Bravo, Andrea

Garren, Melissa

Georgas, Nickitas

Giardino, Alessio

Goldstein, Evan

Gomez-Pujol, Lluis

Gopal, Sucharita

Goring, Derek

Gower, Jim

Guerrero, Massimo

Haffray, Pierrick

Haller, Merrick

Hamsher, Sarah E.

Hansen, Jeffrey

Harris, Daniel

Hirano, Kohin

Hoadley, Kenneth D.

Holt, Teddy

Hook, Sharon E.

Huang, Wenrui

Ikari, Hiroyuki

Irie, Nasayasu

Ivanova, Elena P.

Jacobsen, Karsten

James, Margaret

Jayaratne, Ravindra

Jean-Luc, Menet

Jormalainen, Veijo

Kaksonen, Anna

Kamenov, George

Kampanis, Nikolaos A.
Kämpf, Jochen

Kärnä, Tuomas

Keshtpoor, Mohammad

Khangaonkar, Tarang

Kian, Rozita

Kilibarda, Zoran

Kim, Youngbok

Kitikidou, Kyriaki

Klaucke, Ingo

Kolluru, Venkat

Kooistra, Wiebe H.C.F.

Kovaltchouk, Thibaut

Kristensen, Erik

Kubicki, Adam

Ladrat, Christine Delbarre

LaMontagne, Michael G.

Lara, Javier

Larson, Shawn

Lazakis, Iraklis

Lewis, Matt

Lippert, Tristan

Lisney, Thomas J.

Liu, Yucheng

Lopez, Omar D.

Lottici, Vincenzo

Lueck, Rolf G.

Macleod, Catriona

Maloney, Jillian

Martínez-Guitarte, José-luis

Mase, Hajime

Maspataud, Aurélie

Massey, Chris

Memos, Constantine

Mendelssohn, Irv

Mewis, Peter

Michel, Jaqueline

Missaghi, Shahram

Mizukawa, Hazuki

Moarefdoost, M. Mohsen

Moghimi, Saeed

Moseman-Valtierra, Serena

Moser, Cameron S.

Moser, Gleyci

Motley, Michael R.

Muñoz-Perez, Juan Jose

Muylaert, Koenraad

Nascimbene, Roberto

Nauw, Janine

Neil, Irvine

Nielsen, Morten Holtegaard

O'Connor, Michael

Orvain, Francis 
Paci, Agnese

Palinkas, Cindy

Pandey, Pramod K.

Patterson, Mark

Penta, Bradley

Petersen, Jens Kjerulf

Pineau-Guillou, Lucia

Poizot, Emmanuel

Postacchini, Matteo

Potemra, Jim

Poulin, Francis

Qi, Jianhua

Ravens, Thomas M.

Reikard, Gordon

Riisgaard, Hans Ulrik

Roeber, Volker

Roelvink, Dano

Roncin, Kostia

Ross, Ian

Rovelli, Lorenzo

Rowley, Clark

Rozas, Lawrence P.

Ruhl, Henry A.

Sabzehgar, Reza

Safak, Ilgar

Salta, Maria

Sanford, Robert

Sang, Tzu-Hsien

Santamaria, Carlos

Santos-Echeandía, Juan

Schat, Henk

Schmitt, Thierry

Schultz, Michael P.

Settimo, Gaetano

Shafiee, Mahmood

Shen, Jian

Siegel, Stefan

Signell, Rich

Silva, Ana Nobre

Skarke, Adam

Skov, Martin

Smith, Jane

Smith, William

Son, Seung-Hyun

Steen, Sverre
Stenius, Ivan

Stephenson, Wayne

Stiros, Stathis

Stott, Jeffrey

Strand, Åsa

Suh, SeungWon

Swain, Geoffrey

Szpilka, Christine

Taflanidis, Alexandros

Theuerkauf, Ethan J.

Tim, Le Bas

Tom, Nathan

Tuomi, Laura

Udovydchenkov, Ilya

Urbansky, Ralph

Uriondo, Zigor

Vadas, Sharon

Vallicrosa, Guillem

Van Dorp, Johan René

Vandeputte, Marc

Vargas, Fabio

Vázquez, Juan-Tomás

Viola, Ignazio Maria

Walstra, Dirk-Jan

Wang, Chi-Hsiang

Wang, Hongqing

Wang, Xin

Warner, John

Wase, Nishikant

Weber, Jochem

Wei, Zhangping

White, Helen K.

Wilkinson, Shaun

Wise, John

Wolgamot, Hugh A.

Work, Thierry

$\mathrm{Wu}$, Wencen

Xia, Meng

Xing, Tao

Yang, Xiufeng

Yoon, Hyun-Doug

Yoshida, Hiroshi

Zaron, Edward D.

Zhang,

Joseph 